\title{
Optimization of superconducting critical parameters by tuning the size and magnetization of arrays of magnetic dots
}

\author{
A. V. Silhanek, ${ }^{1, *}$ W. Gillijns, ${ }^{1}$ M. V. Milošević, ${ }^{2,3}$ A. Volodin, ${ }^{1}$ V. V. Moshchalkov, ${ }^{1}$ and F. M. Peeters ${ }^{3, \dagger}$ \\ ${ }^{1}$ INPAC-Institute for Nanoscale Physics and Chemistry, Katholieke Universiteit Leuven, Celestijnenlaan 200 D, \\ B-3001 Leuven, Belgium \\ ${ }^{2}$ Department of Physics, University of Bath, Claverton Down, Bath BA2 7AY, United Kingdom \\ ${ }^{3}$ Department Fysica, Universiteit Antwerpen, Groenenborgerlaan 171, B-2020 Antwerpen, Belgium \\ (Received 18 June 2007; revised manuscript received 19 July 2007; published 10 September 2007)
}

\begin{abstract}
We investigate the nucleation of superconductivity in an Al film deposited on top of an array of micromagnets with perpendicular anisotropy by de transport measurements. The absolute control of the magnetic state of the ferromagnets enabled us to explore the superconductor-normal-metal phase boundary as a function of the magnetization $M$ of the dots. For a given external homogeneous field $H$, the magnetization of the dots can be tuned in such a way to optimize the field and current compensation in the sample, thus yielding a clear enhancement of the upper critical field $H_{c 2}$. In addition, we theoretically demonstrate that the critical current $I_{c}$ for a given temperature could be further enhanced by properly adjusting the size of the magnetic dots. These results are fully corroborated by the Ginzburg-Landau theory as well as analytic estimates.
\end{abstract}

DOI: 10.1103/PhysRevB.76.100502

Controlling the critical parameters of superconductors has been a long pursued objective of condensed matter physicists. ${ }^{1}$ Arguably among the most relevant of these goals is the increase of the critical field below which superconductivity nucleates. For a given material this effect can be achieved by doping the starting compound in such a way that the electronic mean free path $\ell$ becomes smaller than the superconducting coherence length $\xi$ (Ref. 2) or by nanopatterning the material in order to promote surface superconductivity. ${ }^{3}$ Both cases manifest themselves as an enhancement of the upper critical field by a field-independent factor which is determined by either $\ell$ or the geometrical details of the nanostructuring.

An alternative way of preventing the deterioration of the superconducting state under external magnetic fields consists of locally counteracting the applied field by introducing an array of micrometer-sized paramagnets with high magnetic susceptibility. ${ }^{4}$ In this case superconductivity would nucleate in the interstitial position between neighboring paramagnets where the returning stray field of the magnetic elements nearly compensates the external field, ${ }^{5}$ and thus higher fields than in normal conditions could be sustained. ${ }^{6}$ A very close approximation to this situation can be achieved by using a periodic array of ferromagnets with a tunable magnetic moment $\mathbf{m}$ aligned with the applied field $\mathbf{H}$. In this case, unlike for the paramagnets, by changing the polarity of the magnetic moments of the dots one can counteract the external homogeneous field either above ( $\mathbf{m}$ and $\mathbf{H}$ antiparallel) or in between the magnetic elements $\left(\mathbf{m}\right.$ and $\mathbf{H}$ parallel). ${ }^{7}$ These field-compensation effects can lead to a substantial increase of the upper critical field in a field-temperature region determined by the magnetization of the dots.

In this work, we study the field compensation effects in an Al thin film evaporated on top of a square array of $\mathrm{Co} / \mathrm{Pt}$ microdots with tunable out-of-plane magnetization. The selected materials give rise to a sizable difference between the magnetic and superconducting characteristic energies which allowed us to investigate the superconducting phase without perturbing the magnetic state of the dots. We show that by
PACS number(s): 74.78.Fk, 74.25.Dw, 74.25.Fy

adjusting the magnetization of the dot array it is possible to locally compensate the external field in between the dots and thus promote the nucleation of superconductivity at nonzero fields. The highest field at which an optimum compensation can be achieved is only limited by the saturation magnetization of the dots at remanence. As the magnetization of the micromagnets is gradually increased the optimum superconducting critical temperature $T_{c}(H)$ exhibits oscillations similar to those reported in the Little-Parks experiment with cusplike features that can be related to the creation of vortexantivortex pairs by the magnetic dots. Theoretical interpretations and consequences are discussed within the GinzburgLandau formalism and complemented by a partial analytic approach from London theory. The present work complements previous experimental ${ }^{8}$ and theoretical ${ }^{9,10}$ investigations directly addressing the necessary magnetic state of the hybrid system in order to maximize the superconducting critical temperature for different fields.

The sample used for this study is a ferromagnet/insulator/ superconductor $(\mathrm{F} / \mathrm{I} / \mathrm{S})$ trilayer evaporated on a $\mathrm{Si} / \mathrm{SiO}_{2}$ substrate. The $\mathrm{S}$ layer is a 50 -nm-thick $\mathrm{Al}$ film with critical temperature $T_{c 0}=1.321 \mathrm{~K}$ in the as-grown state. Experimentally estimated values of coherence length and penetration depth at $T=0$ are $\xi(0)=117 \mathrm{~nm}$ and $\lambda(0)=98 \mathrm{~nm}$. A 5-nm-thick Si layer separates the $\mathrm{Al}$ film from an underlying square array of circular magnetic dots with lattice spacing $a=2 \mu \mathrm{m}$ [see Fig. 1(a)]. In this way the interaction between the $\mathrm{F}$ and $\mathrm{S}$ layers is purely magnetic and local depletions of the superconducting order parameter arising from electronic coupling can be neglected. The ferromagnetic dots have a diameter of $1.36 \mu \mathrm{m}$ and consist of a 2.5-nm Pt buffer layer covered with a $[0.4-\mathrm{nm} \mathrm{Co} / 1.0-\mathrm{nm} \mathrm{Pt}]_{10}$ multilayer with magnetization perpendicular to the sample surface. ${ }^{11}$

The magnetization $M(H)$ of the $\mathrm{Co} / \mathrm{Pt}$ dot array determined by superconducting quantum interference device (SQUID) magnetometry at $T=5 \mathrm{~K}$ shows a saturation field of $H_{s} \sim 500 \mathrm{mT}$, a coercive field of $70 \mathrm{mT}$, and a remanent magnetization $40 \%$ smaller than the saturation magnetization. After following a degaussing procedure with field steps 
$\delta H \ll \sigma$, where $\sigma$ is the half width at half maximum of the $d M / d H$ vs $H$ curve, it is possible to reduce the remanent magnetization down to $0.5 \%$ of the saturation value. Figure 1(a) shows a magnetic force microscopy image of the $\mathrm{Co} / \mathrm{Pt}$ dots after the demagnetization procedure. In the demagnetized state each dot exhibits a dendriticlike magnetic multidomain structure with minimum lateral size of $100 \mathrm{~nm}$. As detailed in Ref. 8 after demagnetizing the sample it is possible to control the out-of-plane magnetization of the dots by simply limiting the maximum field excursions $H_{m}<H_{s}$. It is worth emphasizing that since the superconducting upper critical field $H_{c 2} \ll H_{m}$ in the whole temperature range, once the magnetic state of the dots is set, they remain nearly unperturbed $^{12}$ during the investigation of the superconducting phase. 8,13

Our theoretical formalism relies upon the GinzburgLandau (GL) equations for the order parameter $\Psi$ and the vector potential $\mathbf{A}$, solved self-consistently. In dimensionless form, when keeping the temperature dependence explicitly, the GL equations become

$$
\begin{gathered}
{\left[(-i \boldsymbol{\nabla}-\mathbf{A})^{2} \Psi=\Psi\left(1-t-|\Psi|^{2}\right)\right],} \\
-\kappa^{2} \boldsymbol{\nabla} \times \boldsymbol{\nabla} \times \mathbf{A}=\operatorname{Im}\left(\psi^{*} \boldsymbol{\nabla} \psi\right)-|\psi|^{2} \mathbf{A},
\end{gathered}
$$

where distances are expressed in units of coherence length $\xi(0)$, A in $H_{c 2}(0) \xi(0), t=T / T_{c}, \Psi$ is normalized by its equilibrium value in the absence of magnetic field, and $\kappa=\lambda / \xi$. For thin superconductors (thickness $<\xi, \lambda$ ), Eqs. (1) and (2) may be averaged over the sample thickness. For details of the numerics, periodic boundary conditions, and the choice of gauge, depending on the applied field and limitations imposed by the virial theorem, ${ }^{14}$ we refer to Ref. 9. The ground-state vortex configurations of all investigated stable states are determined by comparing the Gibbs free energy $\mathcal{F}=V^{-1} \int\left(2\left(\mathbf{A}-\mathbf{A}_{0}\right) \mathbf{j}-|\Psi|^{4}\right) d \mathbf{r},{ }^{16}$ where $\mathbf{j}$ denotes the local supercurrent [right-hand side of Eq. (2)], $\mathbf{A}_{0}$ the applied vector potential, and $V$ the sample volume. On the other hand, the $H$-T superconducting-normal-metal phase boundary is determined by a threshold condition for maximal Cooperpair density in the sample-i.e., $|\Psi|_{\max }^{2}<10^{-12}$. To determine the critical current of the sample, the applied current in the $x$ direction is simulated by adding a constant $A_{c x}$ to the vector potential of the applied magnetic fields. ${ }^{9}$ Note that the current $j_{x}$ in the sample resulting from the applied $A_{c x}$ is obtained after integration of the $x$ component of the induced supercurrents [calculated from Eq. (2)] over the $y=$ cons $t$ cross section of the sample. With increasing $A_{c x}$, the critical current is reached when the stationary solution for Eqs. (1) and (2) can no longer be found [i.e., (anti)vortices are driven in motion by the Lorentz force].

Enhancement of the critical field. Experimental (symbols) and theoretical (lines) $M-T$ boundaries, obtained at different matching fields $H_{n}=n \Phi_{0} / a^{2}$ (with $\Phi_{0}$ the flux quantum) for the above described sample, are shown in Fig. 1(b). The presented theoretical fit was obtained using the exact geometrical parameters from the experiment, but with $\xi(0)=95 \mathrm{~nm}$ and $\lambda(0)=110 \mathrm{~nm}$, which are within $20 \%$ of the experimental estimates. In the demagnetized state $(M=0)$ the superconductor-normal-metal phase boundary $T_{c}(H)$ is in-

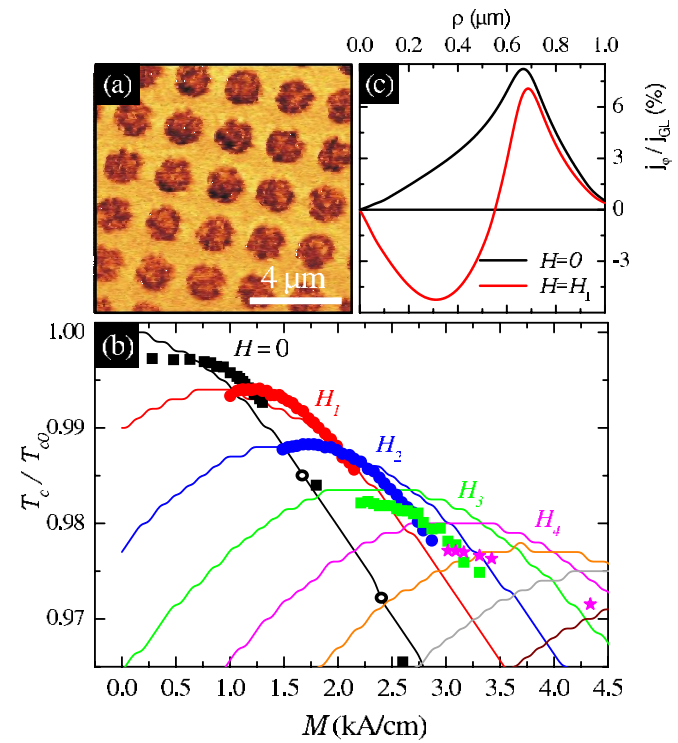

FIG. 1. (Color online) (a) Room-temperature magnetic force microscopy image corresponding to the dot array in the demagnetized state. The horizontal bar indicates a $4-\mu \mathrm{m}$ scale. (b) Critical temperature $T_{c}(M)$ normalized by the value for the as-grown sample, as a function of the average magnetization $M$ of the dots, and several applied matching fields $H=H_{n}$. Solid lines correspond to the theoretical $T_{c}(M)$ at different matching fields $H=H_{n}$ obtained from Ginzburg-Landau formalism. The small ripples observed in the calculated $T_{c}(M)$ are consequence of the chosen temperature step. Open circles on the $H=0$ curve indicate magnetization values for which vortex-antivortex pairs are induced by the magnets. (c) Current profile normalized by the Ginzburg-Landau depairing current $j_{G L}$ in the superconductor above a magnetic dot, for $M=1 \mathrm{kA} / \mathrm{cm}$ and $T=0.992 T_{c}$, without and with a pinned vortex on top.

sensitive to the field polarity; i.e., the $T_{c}(H)$ curve is symmetric around $H=0$ with a maximum critical temperature $T_{c}^{\max }=\max _{H}\left[T_{c}(H)\right]$ located at zero field. ${ }^{17,18}$ After the dots have been magnetized with a small out-of-plane field and then zero-field cooled, $T_{c}^{\max }$ slightly decreases, whereas the field at which $T_{c}^{\max }$ is observed remains the same, $H=0$ [see solid squares in Fig. 1(b)]. This behavior continues until a critical value $M_{0} \sim 1.2 \mathrm{kA} / \mathrm{cm}$ is reached. At this magnetization, the same maximum critical temperature is obtained for both $H=0$ and $H=H_{1}$. The explanation for this phenomenon lies behind the minimization of the supercurrents in the system. Indeed, as illustrated in Fig. 1(c), an identical value for the spatial integral of the total current in a unit cell at fields $H=0$ and $H=H_{1}$ can be reached for this particular $M$. At $H=0$ clockwise screening currents are induced in the film by the magnetic dot, whereas at $H=H_{1}$ the counterclockwise currents of the pinned vortex partially compensate the screening currents, leading to a similar total average current.

As the magnetization is further increased, $T_{c}^{\max }$ shifts to $H=H_{1}$ [solid circles in Fig. 1(b)]. The same trend continues with increasing $M$, with $T_{c}\left(M, H_{n+1}\right)$ becoming gradually higher than $T_{c}\left(M, H_{n}\right)$. In other words, with increasing magnetization of the dots, the critical temperature turns out to be higher for higher applied fields, contrary to the conventional 

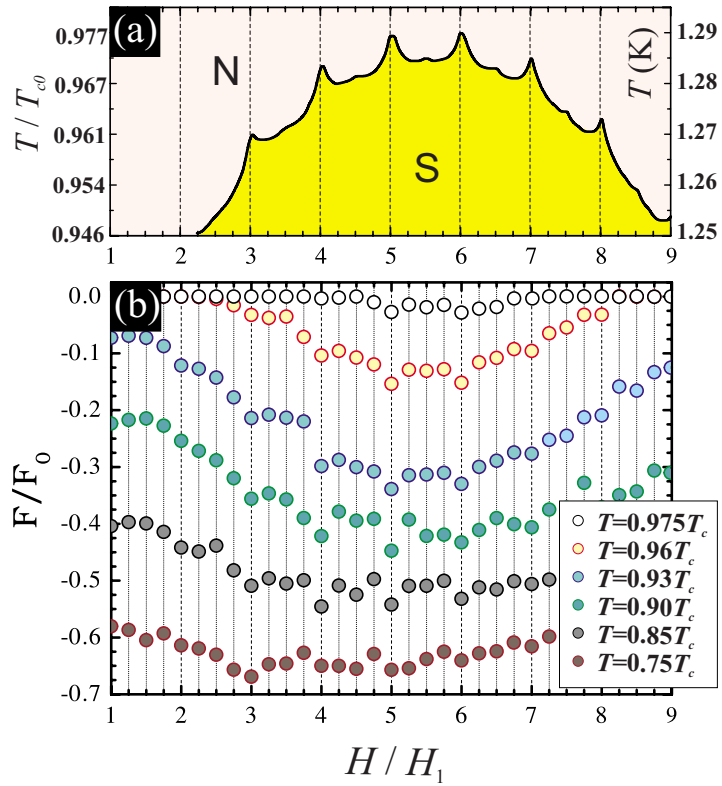

FIG. 2. (Color online) (a) $H-T$ superconducting-normal-metal $(\mathrm{S} / \mathrm{N})$ phase boundary for a magnetization of the dots $M$ $=4.5 \mathrm{kA} / \mathrm{cm}$ (experimental data). (b) Corresponding free energy diagrams vs applied magnetic field (in a field-cooled regime) at different temperatures (from Ginzburg-Landau theory).

behavior. It is important to note that the crossover at $M$ $=M_{0}$ from $T_{c}^{\max }$ located at $H=0$ to $H=H_{1}$ does not coincide with the generation of a vortex-antivortex pair by the magnetic dots. Indeed, the first vortex-antivortex pair appears at a somewhat larger $M$ value [as indicated by open circles on the $H=0$ curve in Fig. 1(b)]. ${ }^{6,9}$

In Fig. 2(a), we show the experimental $H-T$ phase boundary at $M \approx 4.5 \mathrm{kA} / \mathrm{cm}$ for which $T_{c}$ at fields $H_{5}$ and $H_{6}$ nearly coincide. Theoretical results are given in a free energy diagram in Fig. 2(b), where each point is obtained independently, in a "field-cooled" regime. At high temperatures ( $T=0.975 T_{c}$ ) the states for $H=H_{5}$ and $H=H_{6}$ have the lowest energy. Note, however, that with decreasing temperature the most energetically favorable states shift towards lower matching fields. Namely, decreasing temperature hampers the ability of the stray field generated by the magnetic dots to penetrate into the $\mathrm{S}$ layer, as anticipated in Ref. 10. As a consequence, the compensation effects for the enhancement of superconductivity become less efficient.

This scenario is in good agreement with the evolution of the critical current $I_{c}$ as a function of field for different temperatures and $M \sim 1.45 \mathrm{kA} / \mathrm{cm}$, as shown in Fig. 3(a). It can be seen that for $T>1.28 \mathrm{~K}$, the maximal critical current $I_{c}^{\max }$ is located at $H=H_{1}$. However, by decreasing temperature the screening power strengthens and as a consequence $I_{c}^{\max }$ shifts to $H=0$.

In Fig. 3(b) the calculated $I_{c}(M)$ at zero field (dashed line) is directly contrasted with the maximum $I_{c}(M)$ that can be obtained by conveniently setting the external field at different multiples of the matching field $H_{1}$. The $I_{c}(M)$ is calculated within the GL model using the parameters from the experiment and $t=0.97$.

Maximal achievable critical temperature $T_{c}(H)$. From a
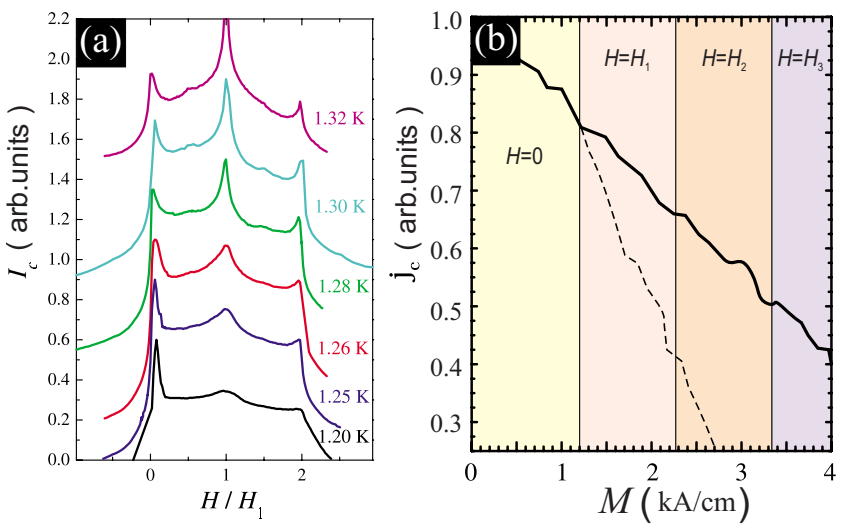

FIG. 3. (Color online) (a) Measured critical current as a function of applied field for the magnetization of the dots $M \approx 1.45 \mathrm{kA} / \mathrm{cm}$ and different temperatures. For the sake of clarity the curves have been displaced vertically. (b) Calculated maximal critical current density $J_{c}^{\max }$ vs $M$ at $t=0.97$, in applied field. Dashed line shows the $I_{c}(M)$ behavior in the absence of external applied field $H$.

more practical point of view, compensation effects can be used in such a way that at every applied homogeneous magnetic field it is possible to adjust the magnetization of the dots in order to maximize $T_{c}(H)$. The result of this procedure is shown in Fig. 4(a) where the evolution of $T_{c}^{\max }(H)$ is obtained by determining at each field the magnetization $M$ necessary to retain the maximum critical temperature. In this figure it can clearly be seen that $T_{c}(H)$ for the dots in the demagnetized state (open symbols) degrades much faster with field than when the dots are adjusted to obtain optimum compensation (solid symbols). As expected, this enhancement of $T_{c}(H)$ becomes more evident for fields above the first matching field-i.e., once vortex-antivortex pairs start to be induced in the sample. These results are consistent with the theoretical estimation (calculated only at the matching fields $H=H_{n}$ ) shown in Fig. 4(b).

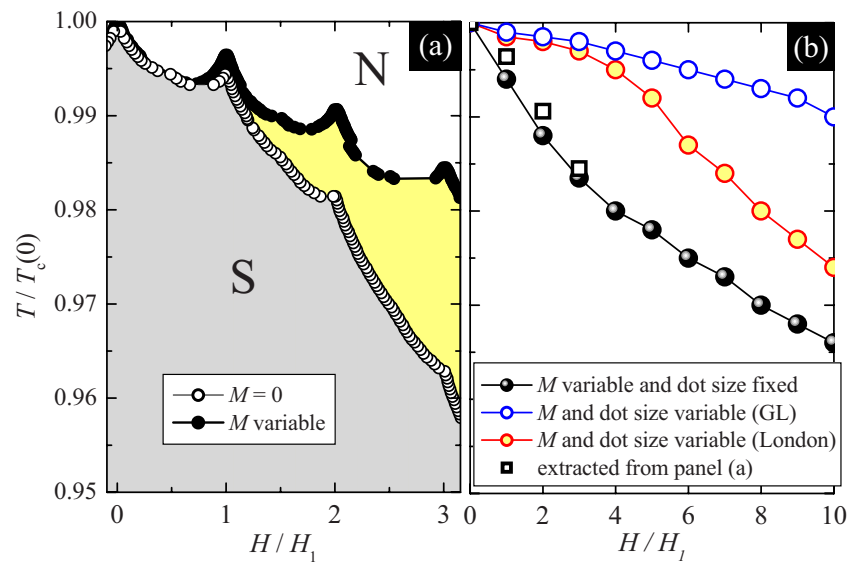

FIG. 4. (Color online) (a) Experimentally determined critical temperature as a function of field for dots in the demagnetized state (open symbols) and for variable $M$ needed to optimize $T_{c}$ (solid symbols). (b) Theoretical simulation of optimized $T_{c}$ for variable $M$ (lower curve) as corresponds to the experiment (open squares). The two upper curves show the theoretical prediction for variable $M$ and sizes of the magnets using GL formalism and using an analytical approximation. 
Interestingly, still a further improvement of the compensation effects can be achieved if the geometry of the magnets is also changed-i.e. their radius and thickness-besides just the magnetization. In such an effort, the screening currents under the magnetic dot can be engineered to match as close as possible (and thus best compensate) the profile of vortex currents for a given vorticity. In principle, for best optimization, the radius of the dots should match the size of the vortex lines at a desired temperature and for lower (larger) vorticity magnets should be made thicker (thinner). The optimal $T_{c}(H)$ obtained from GL theory is shown as the top set of data in Fig. 4(b) and shows an extraordinary enhancement of $T_{c}$ : up to the tenth matching field, the critical temperature of the film has decreased by only $\approx 1 \%$ compared to $T_{c 0}$.

A similar result can be obtained within the London approximation by using the expression of the azimuthal current distribution for a magnet of radius $R$, thickness $D$, and magnetization $M$, placed at a distance $l$ above the superconducting film of thickness $d$ as shown in Ref. 15. Following the approach of Clem, ${ }^{19}$ assuming for the order parameter of a single vortex a trial function $\psi=\rho / \sqrt{\rho^{2}+L r_{v}^{2}}$, where $r_{v}$ is the size of the core of a single vortex, we obtain the following expression for the current of a giant vortex with vorticity $L$ :

$$
j_{\varphi}^{v}(\rho)=-\frac{\Phi_{0}}{2 \pi \lambda^{3}} \frac{\rho K_{1}\left(\sqrt{\rho^{2}+L r_{v}^{2}}\right)}{\sqrt{L} r_{v} K_{1}\left(\sqrt{L} r_{v}\right) \sqrt{\rho^{2}+L r_{v}^{2}}},
$$

where $\rho$ is the radial distance from the center of the vortex and $K_{1}$ denotes the Bessel function. From this equation, when combined with Eq. (13) from Ref. 15, one can estimate the parameters needed to minimize the total current in the superconducting film. When we incorporated these parameters in the GL approach, we obtain the middle set of data shown in Fig. 4(b). This analytical estimation is in good agreement with our numeric calculations for fields below $H$ $=H_{4}$. For higher vorticity, the real current profile of a giant vortex under each of the dots deviates strongly from Eq. (3) and the analytic minimization does not provide a reliable approximation anymore.

In summary, we have demonstrated experimentally the possibility of optimizing the critical parameters of superconducting films by tuning in situ the magnetization of the $\mathrm{Co} / \mathrm{Pt}$ dots. These results were confirmed using GinzburgLandau simulations, which also revealed further possibilities for maximization of $T_{c}$ in applied field by engineering the magnetic dots. We derived an analytic model that provides a reliable way to design the dots for enhancement of $T_{c}$ at low fields. Our guiding principles are generally applicable to any superconducting geometry and have positive consequences on the critical current as well, all being important for prospective superconducting devices.

This work was supported by the K.U. Leuven Research Fund GOA/2004/02, the Flemish Science Foundation (FWOVl), the Belgian Science Policy, the JSPS/ESF-NES program, and the ESF-AQDJJ network. A.V.S. is grateful for the support from the FWO-Vlaanderen. M.V.M. acknowledges support from the EU Marie-Curie Intra-European Program.

*alejandro.silhanek@fys.kuleuven.be

†rancois.peeters@ua.ac.be

${ }^{1}$ V. V. Moshchalkov, V. Bruyndoncx, L. Van Look, M. J. Van Bael, Y. Bruynseraede, and A. Tonomura, in Handbook of Nanostructured Materials and Nanotechnology, edited by H. S. Nalwa (Academic, San Diego, 2000), Vol. 3.

${ }^{2}$ D. Markowitz and L. P. Kadanoff, Phys. Rev. 131, 563 (1963).

${ }^{3}$ D. Saint-James and P. G. de Gennes, Phys. Lett. 7, 306 (1963).

${ }^{4}$ R. P. Cowburn, D. K. Koltsov, A. O. Adeyeye, M. E. Welland, and D. M. Tricker, Phys. Rev. Lett. 83, 1042 (1999).

${ }^{5}$ This effect is a consequence of the well-known property of paramagnets of concentrating flux lines. See, for example, John David Jackson, Classical Electrodynamics, 3rd ed. (Wiley, New York, 1998).

${ }^{6}$ M. Lange, M. J. Van Bael, Y. Bruynseraede, and V. V. Moshchalkov, Phys. Rev. Lett. 90, 197006 (2003).

${ }^{7}$ Z. R. Yang, M. Lange, A. Volodin, R. Szymczak, and V. V. Moshchalkov, Nat. Mater. 3, 793 (2004).

${ }^{8}$ W. Gillijns, A. V. Silhanek, and V. V. Moshchalkov, Phys. Rev. B 74, 220509(R) (2006).

${ }^{9}$ M. V. Milošević and F. M. Peeters, Phys. Rev. Lett. 93, 267006 (2004).

${ }^{10}$ M. V. Milošević and F. M. Peeters, Phys. Rev. Lett. 94, 227001

(2005).

${ }^{11}$ W. B. Zeper, H. W. van Kesteren, B. A. J. Jacobs, J. H. M. Spruit, and P. F. Carcia, J. Appl. Phys. 65, 4971 (1989).

${ }^{12}$ Since the magnetization loop of the dots is not perfectly square shaped, there is a small variation of the magnetization of the dots while exploring the superconducting state. In our sample the relative difference between the magnetization at $+5 \mathrm{mT}$ and $-5 \mathrm{mT}$ amounts to $\sim 5 \%$ of the remanent magnetization.

${ }^{13}$ M. J. Van Bael, L. Van Look, M. Lange, K. Temst, G. Guntherodt, V. V. Moshchalkov, and Y. Bruynseraede, J. Supercond. 14, 355 (2001).

${ }^{14}$ M. M. Doria, J. E. Gubernatis, and D. Rainer, Phys. Rev. B 39, 9573 (1989).

${ }^{15}$ M. V. Milošević and F. M. Peeters, Phys. Rev. B 68, 094510 (2003).

${ }^{16}$ V. A. Schweigert, F. M. Peeters, and P. Singha Deo, Phys. Rev. Lett. 81, 2783 (1998).

${ }^{17}$ J. I. Martin, M. Velez, J. Nogues, and I. K. Schuller, Phys. Rev. Lett. 79, 1929 (1997).

${ }^{18}$ A. T. Fiory, A. F. Hebard, and S. Somekh, Appl. Phys. Lett. 32, 73 (1978)

${ }^{19}$ J. R. Clem, J. Low Temp. Phys. 18, 427 (1975). 